\title{
Rotation matrix from the mean dynamical equator and equinox at J2000.0 to the ICRS
}

\author{
J. L. Hilton ${ }^{1}$ and C. Y. Hohenkerk ${ }^{2}$ \\ 1 US Naval Observatory, 3450 Massachusetts Ave. NW, Washington, DC 20392, USA \\ 2 Her Majesty's Nautical Almanac Office, Space Science \& Technology Dept., Rutherford Appleton Laboratory, Chilton, \\ Didcot OX11 0QX, UK
}

Received 11 April 2003 / Accepted 23 September 2003

\begin{abstract}
Recommendation VII of Resolution A4 of the XXIst General Assembly of the International Astronomical Union (IAU 1992) states, in part, “... that the principal plane of the new conventional celestial reference system be as near as possible to the mean equator at J2000.0 and that the origin in this principal plane be as near as possible to the dynamical equinox of J2000.0, ...” The resulting International Celestial Reference System (ICRS), however, has a small, but significant, offset requiring a rotation matrix. The solutions for the offset between the mean dynamical pole of the Earth at J2000.0 and the pole of the ICRS determined by Lunar Laser Ranging (LLR) and Very Long Baseline Interferometry (VLBI) differ by several $\sigma$. Similarly, two different definitions have traditionally been used for the position of the mean equinox. Which of these poles and equinoxes should be used is application dependent. We have shown how the rotation matrix for the rotation from the mean dynamical equator and equinox at J2000.0 to the ICRS changes depending on the various assumptions made in constructing it.
\end{abstract}

Key words. ephemerides - reference systems

\section{Introduction}

Resolution A4 VII of the XXIst General Assembly of the International Astronomical Union (IAU 1992) recommended the adoption of a quasi-inertial reference system based on the Very Long Baseline Interferometry (VLBI) observations of extragalactic radio sources. This reference system was to be, "... computed initially for the equator and equinox J2000.0 using the best available values of the celestial pole offset with respect to the IAU expressions for precession and nutation ..." The final reference system, the ICRS, was adopted in 1997 in resolution B2 of the XXIIIrd assembly of the IAU (IAU 1997). Since that time, improved measurements have shown that there is a small, but significant, difference between the orientation of the ICRS and that of the best estimate for the mean dynamical equator and equinox of J2000.0 (henceforth J2000.0). The difference between the orientations of the ICRS and J2000.0 amounts to a few tens of milliarcseconds (mas). This difference can be significant for those working at the highest levels of precision. For example, application of precession and nutation to the ICRS rather than J2000.0 leads to additional errors that grow to the same order of magnitude as the difference in orientation between the ICRS and J2000.0 (Wallace, priv. comm. 2002). We have determined the rotation matrix from J2000.0 to the ICRS using the current best estimates for these two reference systems.

\section{Derivation of the rotation matrix}

The coordinate system of J2000.0 can be described using a set of Cartesian coordinate axes, $X_{\mathrm{J} 2000}, Y_{\mathrm{J} 2000}, Z_{\mathrm{J} 2000}$, where the positive $X_{\mathrm{J} 2000}$ axis points towards the equinox, the positive $Y_{\mathrm{J} 2000}$ axis points towards the direction in the equator of the system that is $90^{\circ}$ to the east of the positive $X_{\mathrm{J} 2000}$ axis, and the positive $Z_{\mathrm{J} 2000}$ axis points in the direction $X_{\mathrm{J} 2000} \times Y_{\mathrm{J} 2000}$. Similarly, the ICRS coordinate system can be described using a set of Cartesian coordinate axes, $X_{\text {ICRS }}, Y_{\text {ICRS }}, Z_{\text {ICRS }}$, where the positive $X_{\text {ICRS }}$ axis points towards the origin of the ICRS coordinate system, the positive $Y_{\text {ICRS }}$ axis points towards the direction in the equator of the system that is $90^{\circ}$ to the east of the positive $X_{\text {ICRS }}$ axis, and the positive $Z_{\text {ICRS }}$ axis points in the direction $\mathrm{X}_{\text {ICRS }} \times \mathrm{Y}_{\text {ICRS }}$.

The transformation of a vector $\boldsymbol{x}$ from the J2000.0 coordinate system, $\boldsymbol{x}_{\mathrm{J} 2000}$, to the ICRS, $\boldsymbol{x}_{\mathrm{ICRS}}$, by a rotation matrix $\boldsymbol{R}$ is:

$\boldsymbol{x}_{\mathrm{ICRS}}=\boldsymbol{R} \boldsymbol{x}_{\mathrm{J} 2000}=\boldsymbol{R}_{\psi} \boldsymbol{R}_{\theta} \boldsymbol{R}_{\phi} \boldsymbol{x}_{\mathrm{J} 2000}$

Send offprint requests to: J. L. Hilton, e-mail: jhilton@aa. usno.navy .mil 


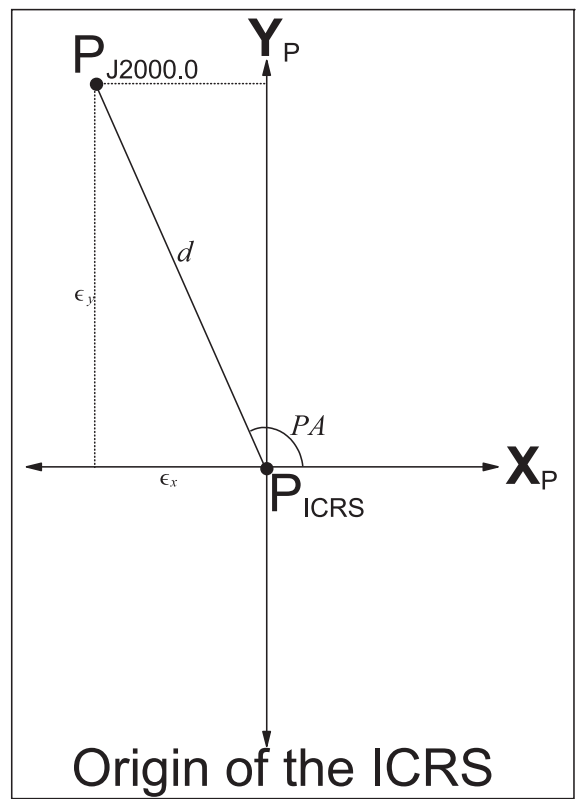

Fig. 1. The position of the pole of $\mathrm{J} 2000.0, \mathrm{P}_{\mathrm{J} 2000.0}$, with respect to the pole of the ICRS, $\mathrm{P}_{\text {ICRS }}$. The relative position is given by the position angle, PA and the distance $d$, or, alternatively by the Cartesian coordinates $\epsilon_{x}$ and $\epsilon_{y}$ where $\mathrm{X}_{\mathrm{P}}$ and $\mathrm{Y}_{\mathrm{P}}$ are the coordinate axes described by Feissel \& Mignard (1998).

where $\boldsymbol{R}_{\psi}, \boldsymbol{R}_{\theta}$, and $\boldsymbol{R}_{\phi}$ are the rotation matrices for the three Eulerian rotations. The Euler angles are: $\psi$, the rotation from the node of the two systems to the origin of the second system around the polar axis of the second system; $\theta$, the inclination of the second system with respect to the first system; and $\phi$, the initial rotation around the polar axis of the first system from its origin to the node of the two systems. The individual Euler rotation matrices are:

$$
\begin{aligned}
\boldsymbol{R}_{\psi} & =\left(\begin{array}{ccc}
\cos \psi & \sin \psi & 0 \\
-\sin \psi & \cos \psi & 0 \\
0 & 0 & 1
\end{array}\right), \\
\boldsymbol{R}_{\theta} & =\left(\begin{array}{ccc}
1 & 0 & 0 \\
0 & \cos \theta & \sin \theta \\
0 & -\sin \theta & \cos \theta
\end{array}\right), \text { and } \\
\boldsymbol{R}_{\phi} & =\left(\begin{array}{ccc}
\cos \phi & \sin \phi & 0 \\
-\sin \phi & \cos \phi & 0 \\
0 & 0 & 1
\end{array}\right) .
\end{aligned}
$$

The difference in position of the $\mathbf{J} 2000.0$ pole with respect to the pole of the ICRS is given as an offset, as published by Feissel $\&$ Mignard (1998) and the International Earth Rotation Service (IERS) (IERS Annual Reports). This offset uses an $X_{P}-Y_{P}$ coordinate system, where the positive $X_{P}$ direction is the $6 \mathrm{hr}$ meridian of the ICRS and the positive $Y_{P}$ direction is the $12 \mathrm{hr}$ meridian, as shown in Fig. 1. Note that this coordinate system is rotated by $90^{\circ}$ from the projection of the ICRS $X_{\text {ICRS }}-Y_{\text {ICRS }}$ plane onto the $X_{P}-Y_{P}$ plane. PA is the position angle, measured from the positive $X_{P}$ axis, of J2000.0 with respect to the ICRS and $d$ is the angular distance between the pole of the ICRS and the pole of J2000.0. Since the $X_{\mathrm{P}}-\mathrm{Y}_{\mathrm{P}}$ plane is rotated with respect to the projection of the $\mathrm{X}_{\text {ICRS }}-\mathrm{Y}_{\text {ICRS }}$ plane, $\alpha_{\text {ICRS }}-\mathrm{PA}=90^{\circ}$ where $\alpha_{\text {ICRS }}$ is the ICRS meridian coordinate ${ }^{1}$. Alternatively, the position of the pole of $\mathrm{J} 2000.0$ with respect to the ICRS can be given in the $\mathrm{X}_{\mathrm{P}}-\mathrm{Y}_{\mathrm{P}}$ plane by the Cartesian coordinates $\epsilon_{x}$ and $\epsilon_{y}$, The relations between the Cartesian and polar coordinates are:

$\epsilon_{x}=\mathrm{d} \cos \mathrm{PA}$ and $\epsilon_{y}=\mathrm{d} \sin \mathrm{PA}$.

The relative positions of the origin of J2000.0 on its equator, and the origin of the ICRS on its equator, are shown in Fig. 2. The origins of both the ICRS and J2000.0 are supposed to coincide with their respective equinoxes. Chapront et al. (2002), however, determined that the actual origin of the ICRS is offset from its equinox by $\Delta o$. The angle $\epsilon_{0}$ is the obliquity of the ecliptic on the ICRS, that is the angle between the ICRS equator and the mean ecliptic as determined by Chapront et al. from LLR observations $\left(23^{\circ} 26^{\prime} 21^{\prime \prime} .41100 \pm 0\right.$ ' $\left.^{\prime} 00005\right)$. The angle $\gamma_{y}$ is the separation between the projection of the node of the mean ecliptic on the ICRS onto the equator of J2000.0 and the equinox of J2000.0 shown in Fig. 2. This angle is given by:

$\tan \gamma_{y}=\tan \Delta \psi \cos \epsilon_{0}$

\footnotetext{
${ }^{1}$ We use the term meridian coordinate instead of right ascension for the longitudinal coordinate of the ICRS because the ICRS is insensitive to the equinox, the traditional origin of right ascension, and its origin is not aligned with the node of its equator on the mean ecliptic.
} 


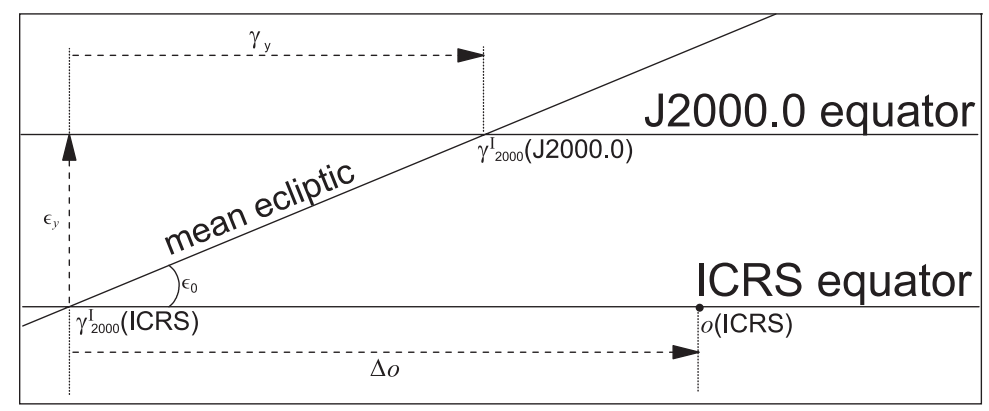

Fig. 2. The relative positions of the origins of the ICRS and J2000.0 at J2000.0. The origin of J2000.0 is the same as the position of the inertial equinox, $\gamma_{2000}^{I}(\mathrm{~J} 2000.0)$. The origin of the ICRS, however, is offset from its inertial equinox, $\gamma_{2000}^{I}$ (ICRS), by $\Delta o$. The projection of the offset between $\gamma_{2000}^{I}\left(\right.$ ICRS) and $\gamma_{2000}^{I}(\mathrm{~J} 2000.0)$ on the ICRS equator is $\gamma_{y}$. And the separation between the two equators at $\gamma_{2000}^{I}$ (ICRS) is the same as the $y$ coordinate of the separation of the two poles, $\epsilon_{y}$. The angle is positive when it goes from left to right or bottom to top in the figure.

Table 1. Recent determinations of the angles between the ICRS and J2000.0.

\begin{tabular}{lllll}
\hline \hline Authors & $\begin{array}{l}\text { Method of } \\
\text { observation }\end{array}$ & $\begin{array}{l}\Delta \epsilon_{0} \\
(\mathrm{mas})\end{array}$ & $\begin{array}{l}\Delta \psi \\
(\mathrm{mas})\end{array}$ & $\begin{array}{l}\Delta o^{a} \\
\text { (mas) }\end{array}$ \\
\hline Chapront et al. (2002) & LLR & $-5.36 \pm 0.10$ & $-44.5 \pm 0.3$ & $55.42 \pm 0.11$ \\
Mathews et al. (2002) & VLBI & -6.8192 & -41.775 & - \\
\hline
\end{tabular}

${ }^{a}$ The angle $\Delta o$ is measured from the node of the ICRS with the mean ecliptic of J2000.0 to the origin of the ICRS. Thus, it has the opposite sign from that published in Chapront et al. (2002), which is measured in the opposite direction (see text for explanation).

Table 2. Rotation parameters between the ICRS and J2000.0.

\begin{tabular}{llllll}
\hline \hline Authors & $\begin{array}{l}\text { Method of } \\
\text { observation }\end{array}$ & $\begin{array}{l}\epsilon_{x} \\
(\mathrm{mas})\end{array}$ & $\begin{array}{l}\epsilon_{y} \\
(\mathrm{mas})\end{array}$ & $\begin{array}{l}\gamma_{y} \\
(\mathrm{mas})\end{array}$ & $\begin{array}{l}\Delta o-\gamma_{y} \\
\text { (mas) }\end{array}$ \\
\hline Chapront et al. (2002) & LLR & $-5.36 \pm 0.54$ & $17.70 \pm 0.20$ & $40.83 \pm 0.46$ & $14.6 \pm 0.5$ \\
Mathews et al. (2002) & VLBI & $-6.819 \pm 0.015$ & $16.6171 \pm 0.0080$ & $38.328 \pm 0.018$ & - \\
\hline
\end{tabular}

where $\Delta \psi$ is the angle between the pole of the ICRS and the pole of J2000.0 as seen from the ecliptic pole. Both $\gamma_{y}$ and $\Delta \psi$ are small angles, so they can be expanded to first order. The error in $\gamma_{y}$ introduced by using the expansions is $\sim 10^{-15}$ radians, much smaller than the uncertainty in $\Delta \psi$ of $10^{-9}-10^{-11}$ radians.

Note that $\gamma_{y}$ is defined such that the initial rotation about the polar axis is from the node of the mean ecliptic on the ICRS to the node of the mean equator of J2000.0, not from the origin of the ICRS to the node of the mean equator of J2000.0. The result is that the values of $\Delta o$ in Table 1 and $\gamma_{y}$ in Table 2 have the opposite signs than they would if they were measured from the origin of the ICRS and mean equinox of J2000.0, respectively.

Using these angles, the three Eulerian rotation angles are then:

$\phi=\mathrm{PA}+\Delta o, \theta=d$, and $\psi=-\mathrm{PA}-\gamma_{y}$.

Simplification of the elements of the rotation matrix by approximating the sine and cosine functions is useful in understanding the rotation between the two coordinate systems.

Expanding $\sin x$ and $\cos x$ to first order implies that, for elements in the rotation matrix that contain sine of a small angle, the uncertainty in that element is $\sigma_{y}^{2} \sim \Sigma 1 \cdot \sigma_{x}^{2}$ while those elements containing only the cosine function have an uncertainty of $\sigma_{y}^{2} \sim \Sigma y^{2} \cdot \sigma_{x}$. The small angles in the rotation matrix are $\sim 10$ mas $\left(5 \times 10^{-8} \mathrm{rad}\right)$ with uncertainties $\sim 0.1 \mathrm{mas}\left(5 \times 10^{-10} \mathrm{rad}\right)$. Thus, the uncertainty of those elements that contain a sine function is $\sim 5 \times 10^{-10}$, and only need to be expanded to first order. On the other hand, those elements that contain only cosine functions have uncertainties $\sim 10^{-18}$ and need to be expanded to second order.

When the values for the angles are substituted into the rotation matrix and the simplifications are carried out, $\boldsymbol{R}$ becomes:

$\boldsymbol{R} \approx\left(\begin{array}{ccc}1-\frac{\left(\Delta o-\gamma_{y}\right)^{2}}{2}-\frac{\epsilon_{y}^{2}}{2} & \Delta o-\gamma_{y} & -\epsilon_{y} \\ -\left(\Delta o-\gamma_{y}\right) & 1-\frac{\left(\Delta o-\gamma_{y}\right)^{2}}{2}-\frac{\epsilon_{y}^{2}}{2} & \epsilon_{x} \\ \epsilon_{y} & -\epsilon_{x} & 1-\frac{\epsilon_{x}^{2}+\epsilon_{y}^{2}}{2}\end{array}\right)$.

The appearance of $\epsilon_{y}$ in the $\boldsymbol{R}_{13}$ and $\boldsymbol{R}_{31}$ elements, $\epsilon_{x}$ in the $\boldsymbol{R}_{23}$ and $\boldsymbol{R}_{32}$ elements and the change in sign between $\epsilon_{x}$ and $\epsilon_{y}$ in the third column and third row appear to be counterintuitive. The reason that these elements appear in this order with these signs is 


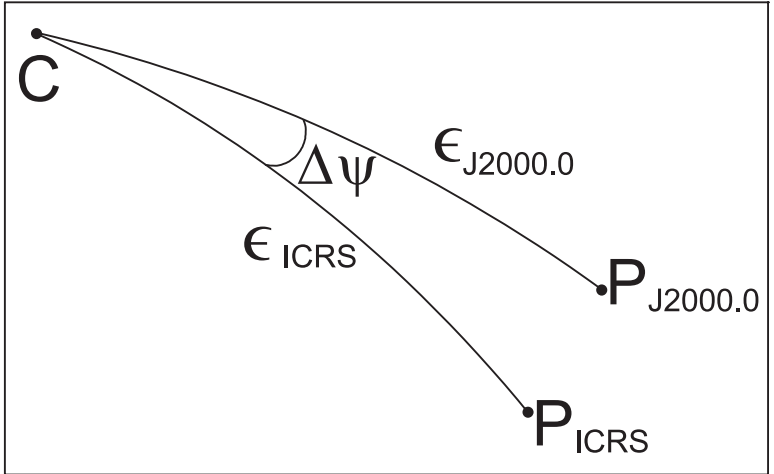

Fig. 3. The position of the poles of the ICRS, $P_{\text {ICRS }}$, and J2000.0, $P_{J 2000.0}$ with respect to the pole of the ecliptic, $C$. The angle between the two poles is $\Delta \psi$ and the difference in their obliquities is $\Delta \epsilon_{0}=\epsilon_{\mathrm{ICRS}}-\epsilon_{J 2000.0}$.

due to the rotation of the $X_{P}-Y_{P}$ plane with respect to the projection of the $X_{I C R S}-Y_{I C R S}$ plane. A position along the positive $X_{P}$ is the equivalent of a position along the positive $Y_{\text {ICRS }}$ axis, while a position along the positive $Y_{P}$ axis is the equivalent of a position along the negative $\mathrm{X}_{\mathrm{ICRS}}$ axis.

Note also, although for parameters $\epsilon_{x}, \epsilon_{y}, \gamma_{y}$, and $\Delta o$ appear in (6) only three of them are independent. The parameter $\gamma_{y}$ is related to $\Delta \psi$ by (4) and $\epsilon_{x}$ and $\epsilon_{y}$ are related to $\Delta \psi$ and $\Delta \epsilon_{0}$, the difference in the obliquity of the ICRS pole and the J2000.0 pole, via (3) and (7) below.

\section{Parameter values}

Mathews et al. (2002) estimate the offset between the pole of J2000.0 and the pole of the ICRS from VLBI observations. The ICRS is also defined by VLBI observations of extragalactic objects (Ma et al. 1998). The relative positions of the two poles, shown in Fig. 3, are given by $\Delta \psi(4)$ and $\Delta \epsilon_{0}$. Mathews et al. do not provide errors in their values; however, other recent determinations of these parameters, such as Shirai \& Fukushima (2001), suggest that the uncertainty is about 0.01 mas for both parameters. This uncertainty will be adopted for the Mathews et al. parameters. From the spherical triangle in Fig. 3, the relations between $\Delta \psi$ and $\Delta \epsilon_{0}$ and the angles $d$ and PA are:

$$
\begin{aligned}
& \epsilon_{\mathrm{J} 2000}=\epsilon_{\mathrm{ICRS}}+\Delta \epsilon_{0} \\
& \cos d=\cos \epsilon_{\mathrm{ICRS}} \cos \epsilon_{\mathrm{J} 2000}+\sin \epsilon_{\mathrm{ICRS}} \sin \epsilon_{\mathrm{J} 2000} \cos \Delta \psi \\
& \sin \mathrm{PA}=\frac{\sin \Delta \psi}{\sin d} \sin \epsilon_{\mathrm{J} 2000} .
\end{aligned}
$$

VLBI, however, is insensitive to the position of the equinox and the origin of the ICRS. The best estimate for these positions is determined by Chapront et al. (2002) from LLR data. Table 1 gives the values for the offsets between J2000.0 and ICRS found by Chapront et al. (LLR) and Mathews et al. (VLBI).

There are at least two possible ways of incorporating the equinox offset into the rotation matrix. The method adopted by the IAU SOFA software and the IERS (Wallace 2002; Capitaine et al. 2003), is to use the offset, $\Delta o$, of the origins purely as determined by LLR and the position of the pole as determined by VLBI observations. The rotation matrix is:

$$
\boldsymbol{R}_{\text {IERS }}=\left(\begin{array}{ccc}
0.99999999999999332 \pm 4 \times 10^{-17} & (7.2 \pm 0.2) \times 10^{-8} & -(8.056 \pm 0.008) \times 10^{-8} \\
-(7.2 \pm 0.2) \times 10^{-8} & 0.99999999999999332 \pm 4 \times 10^{-17} & -(3.306 \pm 0.004) \times 10^{-8} \\
(8.056 \pm 0.008) \times 10^{-8} & (3.306 \pm 0.004) \times 10^{-8} & 0.999999999999996208 \pm 4 \times 10^{-18}
\end{array}\right) .
$$

Aside from the possible systematic error caused by the misalignment of the origin, the accuracy in the rotated position varies from about 0.5 mas near the equator to about 0.02 mas near the poles The variation in the accuracy is caused by the much lower accuracy of the separation between origin of the ICRS and the mean equinox of J2000.0 when compared to the accuracy of the separation between the poles.

An alternative method is to use the value of $\Delta o$ found from the LLR observations with the VLBI value for $\gamma_{y}$ to give $\Delta o-\gamma_{y}$. This method assumes that the offset of the ICRS origin from the ICRS equinox is the same in both methods. VLBI observations, like LLR observations, take place in an inertial framework; thus, the value of $\gamma_{y}$ determined from VLBI observations assumes an inertial mean equinox. The value of $\Delta o-\gamma_{y}$ is then $(55.42 \pm 0.11)-(38.33 \pm 0.02)=17.09 \pm 0.11$ mas in comparison to the Chapront et al. value of $14.6 \pm 0.5$ mas used in deriving (8). Thus,

$\boldsymbol{R}_{12}=-\boldsymbol{R}_{21}=(8.29 \pm 0.05) \times 10^{-8}$.

The difference between the Mathews et al. VLBI-based parameters and the Chapront et al. LLR-based parameters means that there is a possible inconsistency in the $\boldsymbol{R}_{12}$ and $\boldsymbol{R}_{21}$ elements of the rotation matrix that could leave a misalignment in the rotated 
origin of up to 3 mas. Thus, the user also has to be aware that there may be a misalignment of up to 3 mas in the rotated origin, because the method of determining the position of the origin is not wholly consistent with that of determining the position of the pole.

On the other hand, the node of the mean ecliptic in the ICRS, $\gamma_{2000}^{I}(\mathrm{ICRS})$, and the equinox, $\gamma_{2000}^{I}(\mathrm{~J} 2000.0)$, shown in Fig. 2 are specifically for the inertial definition of the ecliptic as described in Standish (1981). The inertial definition of the mean equinox is the one that appears in the IERS Conventions for 2000 (IERS, 2003). However, older ephemerides, such as DE200 are aligned to the rotating definition of the mean equinox Folkner et al (1994). Standish (1981) found a difference in the position of the rotating minus inertial ecliptic of -93.66 mas at J2000.0. Both the $78 \pm 10$ mas counterclockwise offset of the rotating equinox of the DE200 ephemerides from the origin of the ICRS found by Folkner et al., and the $14.6 \pm 0.5$ mas clockwise offset of the inertial equinox found by Chapront et al. are consistent with Standish's estimate. Although Standish also found a difference in the rotating minus inertial obliquity of 3.34 mas at J2000.0, this does not affect the relative position of the pole of J2000.0 with respect to the pole of the ICRS, as the difference in obliquity is caused solely by a change in the position of the pole of the mean ecliptic. Thus, the sole effect of using the rotating definition of the mean equinox rather than the inertial definition is to change the value of $\Delta o$ to

$\Delta o=55.42-93.66=-38.24$ mas.

Thus, the $\boldsymbol{R}_{12}$ and $\boldsymbol{R}_{21}$ elements to become:

$\boldsymbol{R}_{12}=-\boldsymbol{R}_{21}=-(38.3 \pm 0.2) \times 10^{-8}$

if both $\Delta o$ and $\gamma_{y}$ are determined by LLR. If $\Delta o$ is determined by LLR and $\gamma_{y}$ is determined from VLBI then

$\boldsymbol{R}_{12}=-\boldsymbol{R}_{21}=-(37.1 \pm 0.2) \times 10^{-8}$.

The source of this difference is the same possible 3 mas misalignment of the equinox caused by the difference in the VLBI and LLR solutions for $\gamma_{y}$ that causes the difference between (9) and the $\boldsymbol{R}_{12}$ and $\boldsymbol{R}_{21}$ elements in (8). Note also that the signs of the $\boldsymbol{R}_{12}$ and $\boldsymbol{R}_{21}$ elements change indicating that the rotating equinox is on the opposite side of the origin of the ICRS from the inertial equinox.

The uncertainty in the position of the rotating mean equinox with respect to the inertial mean equinox is assumed to be the same as the uncertainty of the frame tie between DE405 and the ICRS of 1 mas (Standish 1998). Thus, the accuracy of the rotation using the rotating definition of the mean equinox is approximately 1.2 mas.

Chapront et al. (2002) also determined values for $\Delta \psi$ and $\Delta \epsilon_{0}$, but the uncertainty in their values is about an order of magnitude greater than those found from VLBI. Note that in Table 1 the orientation of the J2000.0 pole with respect to the ICRS pole found by LLR is significantly different than that found by VLBI. The rotation parameters derived from Chapront et al. and Mathews et al. are given in Table 2 .

The rotation matrix from J2000.0 to the ICRS based on the Chapront et al. (LLR) parameters is:

$$
\boldsymbol{R}_{\mathrm{LLR}}=\left(\begin{array}{ccc}
0.9999999999999938 \pm 2 \times 10^{-16} & (7.1 \pm 0.2) \times 10^{-8} & -(8.6 \pm 0.3) \times 10^{-8} \\
-(7.1 \pm 0.2) \times 10^{-8} & 0.9999999999999938 \pm 2 \times 10^{-16} & -(2.6 \pm 0.1) \times 10^{-8} \\
(8.6 \pm 0.3) \times 10^{-8} & (2.6 \pm 0.1) \times 10^{-8} & 0.99999999999999598 \pm 5 \times 10^{-17}
\end{array}\right) \text {. }
$$

This rotation matrix has the advantage of being determined from a wholly consistent set of parameters. Its accuracy is limited by the accuracy of the LLR observations. Thus, the resulting position after rotation should be accurate to approximately 0.9 mas.

Table 3 presents the angles $\epsilon_{x}, \epsilon_{y}, \gamma_{y}$, and $\Delta o$ for use with (6), for determining each of the five rotation matrices above.

\section{Conclusions}

The orientation of ICRS, as stipulated in Recommendation VII of Resolution A4 of the XXI General Assembly of the International Astronomical Union, was to be aligned as closely as possible with the mean celestial ephemeris pole and equinox of J2000.0. Since its inception, however, better observations have shown a small but significant offset between the ICRS and the mean equator and equinox of J2000.0. The parameters that define this offset have been determined by Chapront et al. (2002) using LLR observations, and Mathews et al. (2002) from VLBI data. These two sets of parameters are significantly different, and the VLBI data are insensitive to the position of the equinox and the origin of the ICRS.

There are also two different definitions for the mean equinox of J2000.0 (Standish 1981), one defined using an inertial ecliptic and the other based on the rotating ecliptic. The IAU (1991) recommended the adoption of the rotating definition, while the IERS (2003) used the inertial definition in setting up the ICRS.

We have thus determined five scenarios to transform positions from J2000.0 to the ICRS. The parameters of which are summarized below and listed in Table 3.

The matrix $\boldsymbol{R}_{\text {IERS }}$, (8), uses an offset of the origin of the ICRS from the mean inertial equinox of J2000.0 determined wholly from LLR data and an offset for the poles determined wholly from VLBI data. This matrix should be used by those who want to produce a rotation that is as close as possible to the positions determined by the IERS. 
Table 3. Offset angles for each of the rotation matrices.

\begin{tabular}{lllllllll}
\hline \hline \multicolumn{3}{c}{ Matrix parameters } & & & $\epsilon_{x}$ & $\epsilon_{y}$ & $\gamma_{y}$ & $\Delta o$ \\
Equinox & $\epsilon_{x}, \epsilon_{y}$ & $\gamma_{y}$ & $\Delta o$ & Name & $(\mathrm{mas})$ & $\begin{array}{l}\Delta o \\
(\mathrm{mas})\end{array}$ & $\begin{array}{l}\text { (mas) } \\
(\mathrm{mas})\end{array}$ \\
\hline 1. Inertial & VLBI & LLR & LLR & $\boldsymbol{R}_{\text {IERS }}$ & -6.819 & 16.6171 & 40.83 & 55.42 \\
2. Inertial & VLBI & VLBI & LLR & & -6.819 & 16.6171 & 38.328 & 55.42 \\
3. Rotating & VLBI & LLR & LLR & & -6.819 & 16.6171 & 40.83 & -38.24 \\
4. Rotating & VLBI & VLBI & LLR & & -6.819 & 16.6171 & 38.328 & -38.24 \\
5. Inertial & LLR & LLR & LLR & $\boldsymbol{R}_{\text {LLR }}$ & -5.36 & 17.7 & 40.83 & 55.42 \\
\hline
\end{tabular}

Alternatively, the separation, $\gamma_{y}$, between the projection of the equinox onto the equator of the ICRS and the node of the ecliptic on the equator of the ICRS may be determined from the VLBI offset of the pole. This value can then be combined with the offset between the equinox on the ICRS and the origin of the ICRS found by Chapront et al. from LLR observations. This change in the derivation of $\gamma_{y}$ only affects the $\boldsymbol{R}_{12}$ and $\boldsymbol{R}_{21}$ elements of the rotation matrix as given in (9).

Similarly, using the rotating definition of the mean ecliptic will also change the position of the mean equinox relative to the origin of the ICRS. As with the determination of $\gamma_{y}$, the use of the rotating definition of the equinox only affects the $\boldsymbol{R}_{12}$ and $\boldsymbol{R}_{21}$ elements of the rotation matrix. Equation (11) gives the values of these elements using the rotating definition of the equinox with $\gamma_{y}$ determined from LLR observations, while (12) gives the value for these two elements with $\gamma_{y}$ determined from VLBI observations.

Finally, the matrix $\boldsymbol{R}_{\mathrm{LLR}}$, (13), is based solely upon the parameters determined solely from LLR data by Chapront et al. This matrix should be used by those who desire a rotation based upon an entirely self-consistent set of parameters using the inertial definition of the mean equinox.

Users needing accuracy better than a few tens of mas will need to use one of these matrices for converting from J2000.0 to the ICRS and the subsequent processes of applying precession, nutation, and the formation of an apparent place. However, the user needs to realize that there are historically two different definitions of the mean equinox that differ by 93.66 mas. Finally, the difference in the VLBI and LLR solutions for the position of the mean pole of J2000.0 implies that there may be an inconsistency in determining the equinox that can lead to a misalignment in the origin of up to 3 mas. Thus, care must be used in selecting the proper matrix to rotate between the ICRS and the mean dynamical equator and equinox of J2000.0.

\section{References}

Capitaine, N., Chapront, J., Lambert, S., \& Wallace, P. T. 2003, A\&A, 400, 1145

Chapront, J., Chapront-Touzé, M., \& Francou, G. 2002, A\&A, 387, 700

Feissel, M., \& Mignard, F. 1998, A\&A, 331, L33

Folkner, W. M., Charlot, P., Finger, M. H., et al. 1994, A\&A, 287, 279

IAU 1992, Transactions of the International Astronomical Union, vol. XXIB, 4344

IAU 1997, Transactions of the International Astronomical Union, Vol. XXIIIB, 39

IERS 2003, IERS Conventions 2000, ed. D. D. McCarthy, in preparation

Ma, C., Arias, E. F., Eubanks, T. M., et al. 1998, AJ, 116, 516

Mathews, P. M., Herring, T. A., \& Buffett, B. A. 2002, J. Geophys. Res., 107, B4, 10.1029/2001JB00390

Shirai, T., \& Fukushima, T. 2001, AJ, 121, 3270

Standish, E. M., Jr. 1981, A\&A, 101, L17

Standish, E. M., Jr. 1998, Jet Propulsion Laboratory, Interoffice Memorandum, IOM 312.F98048

Wallace, P. 2002, in Highlights in Astronomy, ed. H. Rickman (San Francisco: Astronomical Society of the Pacific), 12, 128 